\title{
Splenic T1-mapping: a novel quantitative method for assessing adenosine stress adequacy for cardiovascular magnetic resonance
}

Alexander Liu', Rohan S. Wijesurendra', Rina Ariga', Masliza Mahmod', Eylem Levelt ${ }^{1}$, Andreas Greiser², Mario Petrou ${ }^{3}$, George Krasopoulos ${ }^{3}$, John C. Forfar ${ }^{4}$, Rajesh K. Kharbanda ${ }^{4,5}$, Keith M. Channon ${ }^{5}$, Stefan Neubauer ${ }^{1}$, Stefan K. Piechnik ${ }^{1}$ and Vanessa M. Ferreira ${ }^{1 *}$

\begin{abstract}
Background: Perfusion cardiovascular magnetic resonance (CMR) performed with inadequate adenosine stress leads to false-negative results and suboptimal clinical management. The recently proposed marker of adequate stress, the "splenic switch-off" sign, detects splenic blood flow attenuation during stress perfusion (spleen appears dark), but can only be assessed after gadolinium first-pass, when it is too late to optimize the stress response. Reduction in splenic blood volume during adenosine stress is expected to shorten native splenic T1, which may predict splenic switch-off without the need for gadolinium.

Methods: Two-hundred and twelve subjects underwent adenosine stress CMR: 1.5 T ( $n=104 ; 75$ patients, 29 healthy controls); $3 \mathrm{~T}(n=108 ; 86$ patients, 22 healthy controls). Native T1 spleen was assessed using heart-rateindependent ShMOLLI prototype sequence at rest and during adenosine stress $(140 \mathrm{\mu g} / \mathrm{kg} / \mathrm{min}, 4 \mathrm{~min}, \mathrm{IV})$ in 3 short-axis slices (basal, mid-ventricular, apical). This was compared with changes in peak splenic perfusion signal intensity $\left(\Delta \mathrm{S} \mathrm{I}_{\text {spleen }}\right)$ and the "splenic switch-off" sign on conventional stress/rest gadolinium perfusion imaging. $\mathrm{T} 1_{\text {spleen }}$ values were obtained blinded to perfusion $\Delta \mathrm{SI}_{\text {spleen, }}$, both were derived using regions of interest carefully placed to avoid artefacts and partial-volume effects.
\end{abstract}

Results: Normal resting splenic T1 values were $1102 \pm 66 \mathrm{~ms}$ (1.5 T) and $1352 \pm 114 \mathrm{~ms}(3 \mathrm{~T})$, slightly higher than in patients (1083 $\pm 59 \mathrm{~ms}, p=0.04 ; 1295 \pm 105 \mathrm{~ms}, p=0.01$, respectively). $T 1_{\text {spleen }}$ decreased significantly during adenosine stress (mean $\Delta T 1_{\text {spleen }} \sim-40 \mathrm{~ms}$ ), independent of field strength, age, gender, and cardiovascular diseases. While $\Delta T 1_{\text {spleen }}$ correlated strongly with $\Delta \mathrm{SI}_{\text {spleen }}$ (rho $=0.70, p<0.0001$ ); neither indices showed significant correlations with conventional hemodynamic markers (rate pressure product) during stress. By ROC analysis, a $\Delta T 1_{\text {spleen }}$ threshold of $\geq-30 \mathrm{~ms}$ during stress predicted the "splenic switch-off" sign (AUC 0.90, $p<0.0001)$ with sensitivity $(90 \%)$, specificity (88\%), accuracy (90\%), PPV (98\%), NPV (42\%).

Conclusions: Adenosine stress and rest splenic T1-mapping is a novel method for assessing stress responses, independent of conventional hemodynamic parameters. It enables prediction of the visual "splenic switch-off" sign without the need for gadolinium, and correlates well to changes in splenic signal intensity during stress/rest perfusion imaging. $\Delta T 1_{\text {spleen }}$ holds promise to facilitate optimization of stress responses before gadolinium first-pass perfusion CMR.

Keywords: Cardiovascular magnetic resonance, Adenosine stress, Splenic T1, Switch-off, ShMOLLI

\footnotetext{
* Correspondence: Vanessa.ferreira@cardiov.ox.ac.uk

'Oxford Centre for Clinical Magnetic Resonance Research (OCMR), Division of

Cardiovascular Medicine, Radcliffe Department of Medicine, University of

Oxford, Oxford, UK

Full list of author information is available at the end of the article
} 


\section{Background}

Adenosine stress perfusion cardiovascular magnetic resonance (CMR) accurately detects myocardial ischemia and guides clinical decision-making $[1,2]$. However, perfusion CMR has a reported false-negative rate of between 5 and 16\% [2-4], which may lead to suboptimal management strategies. In the absence of poor image quality, inadequate adenosine stress response is the commonest cause of false-negative perfusion scans [4], because conventional hemodynamic markers of stress response, such as heart rate and systolic blood pressure, are unreliable predictors of myocardial vasodilatation and the achievement of maximal hyperemia [5].

Recently, the "splenic switch-off" sign was proposed as a CMR marker of adequate adenosine stress. It describes visually reduced splenic perfusion during stress imaging (spleen appears dark) compared to rest imaging (spleen appears bright) [6], and in retrospective analyses, failed splenic switch-off was more commonly observed in false-negative perfusion scans than true-negatives [6]. The physiological basis for this phenomenon is that splenic blood volume reduces significantly during exercise, due to splanchnic blood redistribution [7, 8], and can manifest as splenic "disappearance" on nuclear imaging [9]. The degree of splenic blood volume reduction is proportional to exercise workload [7], independent of cardiac output [7], and is related to adenosine-mediated splenic vasoconstriction $[10,11]$. More recently, splenic switch-off has been shown to relate to higher myocardial T2 values during dipyridamole stress, further suggesting a connection between splenic and myocardial vascular biology [12].

A key limitation of splenic switch-off is that it can only be assessed after gadolinium first-pass perfusion imaging [6], at which point it is too late to optimize stress adequacy [13]. Repetition of inadequately stressed images would require a wait-period (10-15 $\mathrm{min}$ ) for gadolinium "wash-out" from the LV cavity to optimize myocardialblood contrast during the subsequent (no longer firstpass) stress perfusion imaging, leading to longer scan durations, and exposes patients to additional adenosine and contrast agents [6]. Therefore, a method which can determine stress adequacy and offer opportunities for pre-emptive stress response optimization before gadolinium first-pass perfusion imaging is highly desirable.

Native T1-mapping enables quantitative characterization of tissue blood volumes without the need for gadoliniumbased contrast agents (GBCA) [14-16], and offers the potential to assess stress responses before GBCA first-pass perfusion. T1 (proton spin-lattice) relaxation time is a magnetic property of tissues measured in milliseconds [14], and each tissue type, including the spleen, has its own normal range of $\mathrm{T} 1$ values [14]. $\mathrm{T} 1$ is sensitive to changes in tissue water content or blood volume [15-19], and we recently showed that normal myocardial T1 increases by $6 \%$ during adenosine vasodilatory stress, due to expansions in myocardial blood volume $[15,16]$. Furthermore, stress-T1 appears sensitive to changes in normal, ischemic and infarcted myocardium, without the need for GBCA [15]. Contrary to its vasodilatory effects in the myocardium, adenosine causes splenic vasoconstriction, reducing the splenic blood volume, and thus expected to lower the splenic $\mathrm{T} 1\left(\mathrm{~T} 1_{\text {spleen }}\right)$. Conveniently, the spleen is typically visible on stress perfusion CMR and can be inspected without additional planning.

This study sought to evaluate stress and rest $\mathrm{T} 1_{\text {spleen }}$ as a gadolinium-free CMR marker of adenosine stress responses by comparing with the existing "splenic switch-off" sign and hemodynamic markers. We hypothesized that: (i) $\mathrm{T} 1_{\text {spleen }}$ will decrease significantly from resting values during adenosine stress, due to splenic blood volume reductions and; (ii) stress-related changes in $\mathrm{T} 1_{\text {spleen }}\left(\Delta \mathrm{T} 1_{\text {spleen }}\right)$ correlate to changes in splenic perfusion on CMR (the "splenic switch-off" sign), but without the need for GBCA.

\section{Methods}

All study procedures received favourable opinions from local ethics committees, and all subjects gave written informed consent.

\section{Study population}

To establish the relationship between $\mathrm{T} 1_{\text {spleen }}$ and splenic perfusion/switch-off, retrospective analysis was performed on CMR scans of 212 subjects; 104 subjects had CMR at $1.5 \mathrm{~T}$ (Magnetom Avanto, Siemens Healthcare, Erlangen, Germany) and 108 subjects had CMR at $3 \mathrm{~T}$ (Magnetom Trio a Tim system, Siemens Healthcare, Erlangen, Germany). The $1.5 \mathrm{~T}$ population had 75 patients $(n=36$ known coronary artery disease $[\mathrm{CAD}], n=39$ Atrial Fibrillation $[\mathrm{AF}]$ ) and 29 healthy controls; the $3 \mathrm{~T}$ population had 86 patients $(n=$ 22 known CAD, $n=23$ Type II Diabetes Mellitus [DM], $n=$ 21 Severe Aortic Stenosis [AS], $n=20$ Hypertrophic Cardiomyopathy $[\mathrm{HCM}]$ ) and 22 healthy controls. Healthy controls had no history of cardiovascular disease, were not on regular medications, and had normal electrocardiograms.

\section{CMR protocol}

All subjects avoided adenosine antagonizers (e.g. caffeine) for $\geq 24 \mathrm{~h}$ before CMR. T1-mapping was performed using the Shortened Modified Look-Locker Inversion recovery (ShMOLLI) prototype sequence (WIP 561 and 448C) with inline map generation, which uses 9-heartbeats breathholds per T1-map acquisition and enables on-screen image reconstruction within $10 \mathrm{~s} \mathrm{[14].}$

Native T1-maps were acquired at rest and during peak adenosine stress $(140 \mu \mathrm{g} / \mathrm{kg} / \mathrm{min}, 4 \mathrm{~min}, \mathrm{IV})$ in short-axis (basal, mid-ventricular, apical) slices, followed immediately 
by first-pass perfusion imaging on matching slices during peak stress, with an IV bolus of GBCA $(0.03 \mathrm{mmol} / \mathrm{kg}$ at $6 \mathrm{ml} / \mathrm{s}$; Dotarem, Guerbet, Villepinte, France) and saline flush $(15 \mathrm{ml}$ at $6 \mathrm{ml} / \mathrm{s})[15,16]$. Matching rest perfusion images were acquired $>15$ min after stress perfusion and adenosine discontinuation to allow sufficient time for contrast washout $[15,16]$.

\section{T1-mapping analysis}

Separate data files containing all T1-maps were created and anonymized before analysis by an observer (>3 years of T1-mapping analysis experience) blinded to perfusion images and clinical information. T1-maps were excluded from analysis if the spleen was not clearly visible (2\%), had respiratory-motion artefacts on raw Inversion-Recovery-weighted images $(3 \%)$ or had suboptimal goodness-of-fit $\mathrm{R}^{2}$-maps $(2 \%)[17,20]$. Overall, 738 T1-maps were included in final analysis, using dedicated in-house software MC-ROI (programmed by S.K.P. in IDL, version 6.1, Exelis Visual Information Solutions, Boulder, Colorado) [14-18, 20]. To estimate mean native $\mathrm{T} 1_{\text {spleen, }}$ regions of interest (ROIs) were manually placed on T1-maps to include as much splenic tissue as possible, avoiding partial volume effects from large splenic blood vessels and borders with neighbouring tissues (Fig. 1). ROIs were quality checked against corresponding InversionRecovery-weighted images and $\mathrm{R}^{2}$-maps. To derive thresholds suitable for direct application on the CMR console, splenic T1-reactivity to adenosine stress $\left(\Delta \mathrm{T} 1_{\text {spleen }}\right)$ was expressed in absolute terms: $\Delta \mathrm{T} 1_{\text {spleen }}$ $(\mathrm{ms})=$ Stress $\mathrm{T} 1_{\text {spleen }}-$ Rest $\mathrm{T} 1_{\text {spleen }}$.

\section{$\mathrm{T} 1_{\text {spleen }}$ intra-scan variability assessments}

Inter-slice variability in resting $\mathrm{T} 1_{\text {spleen }}$, stress $\mathrm{T} 1_{\text {spleen }}$ and $\Delta \mathrm{T} 1_{\text {spleen }}$ were assessed in cases where matching stress and rest T1-maps were performed in $\geq 2$ different short-axis slice positions. To assess for intra-slice $\mathrm{T} 1_{\text {spleen }}$ variability, we re-analyzed healthy-volunteers data from the original ShMOLLI methods paper, where T1-maps were repeated $>15 \mathrm{~min}$ apart in the same short-axis slice within the same scan [14]. Intra-scan variability was calculated as the standard deviation of differences-from-the-mean in each individual.

\section{Splenic perfusion analysis}

Splenic first-pass perfusion was analysed by an observer ( $>4$ years of perfusion imaging analysis experience) blinded to T1-maps and clinical information, using $\mathrm{CMR}^{42}$ software (Circle Cardiovascular Imaging Inc., Calgary, Canada). Splenic ROIs were placed on stress and rest perfusion images with frame-by-frame manual

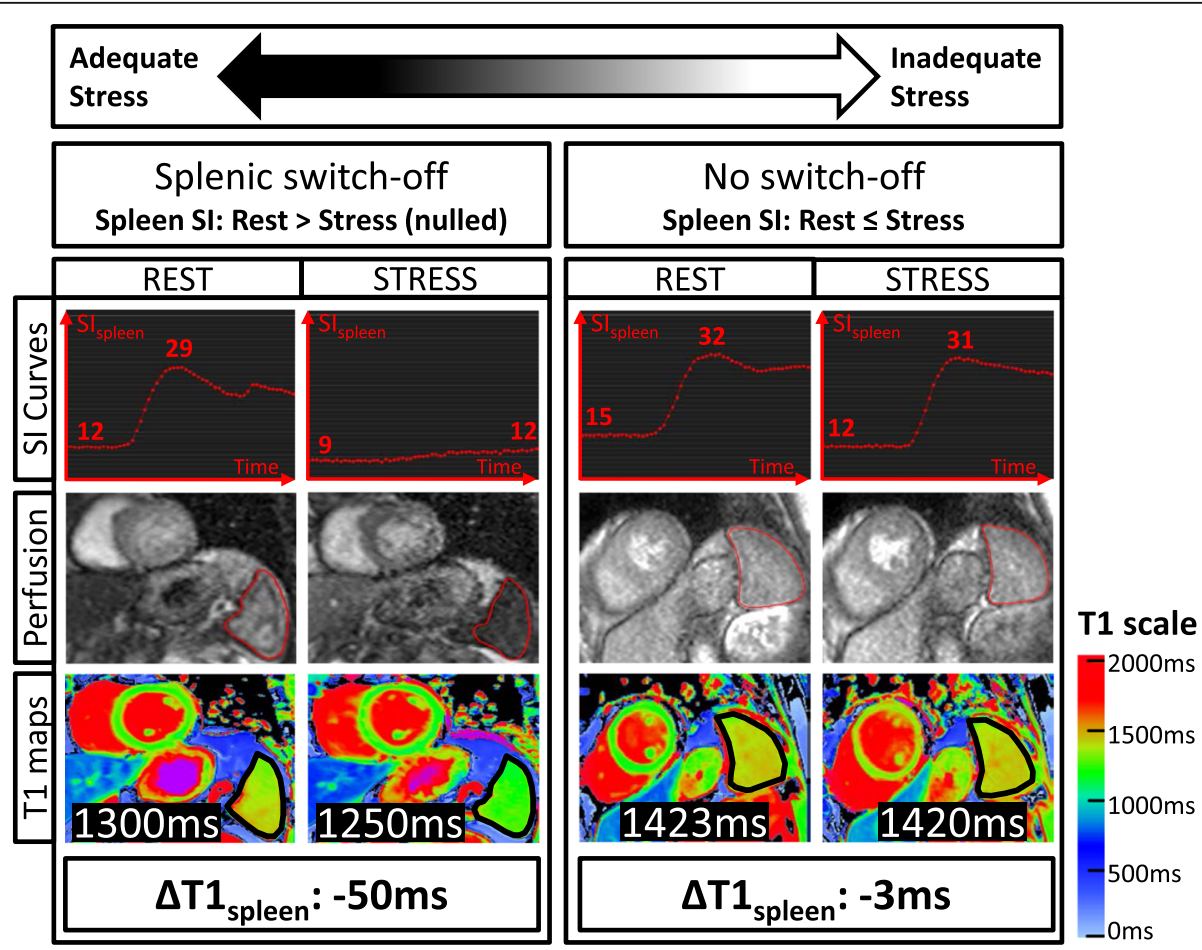

Fig. 1 Representative stress and rest splenic first-pass gadolinium perfusion and native T1-maps. Signal intensity (SI) curves represent splenic perfusion SI (y-axis, arbitrary units) over time ( $x$-axis, 50-60 s). The maximum and minimum SIspleen are as indicated. Splenic regions of interests on perfusion images and T1-maps are outlined in red and black, respectively. Mean native $T 1_{\text {spleen }}$ and stress changes $\left(\Delta T 1_{\text {spleen }}\right)$ are as labelled. $3 T$ images were used for illustration (observed $\Delta T 1_{\text {spleen }}$ and $\Delta S I_{\text {spleen }}$ are field strength independent) 
correction for artefacts and respiratory motion, to generate curves showing mean splenic signal intensity (SI, arbitrary units) changes over time (50-60s). Peak splenic perfusion $\mathrm{SI}\left(\mathrm{SI}_{\text {spleen }}\right)$ was estimated as the numerical difference between baseline-SI and maximal-SI during splenic first-pass perfusion as previously described [6]. Adenosine-induced changes in $\mathrm{SI}_{\text {spleen }}$ compared to rest were expressed in percentages: $\Delta \mathrm{SI}_{\text {spleen }}$ $(\%)=\left(\right.$ StressSI $\left._{\text {spleen }}-\operatorname{RestSI}_{\text {spleen }}\right) \div$ RestSI $_{\text {spleen }} \times 100 \%$.

Splenic switch-off on perfusion imaging was visually assessed by 2 independent observers ( $>3$ years clinical CMR perfusion experience). In the $5 / 212$ cases where the 2 observers disagreed, adjudication was sought from a $3^{\text {rd }}$ independent observer (Fig. 1). Perfusion images were graded as previously described [6]: either displaying splenic switch-off (the spleen on rest imaging is clearly brighter than on stress imaging), or no switch-off (the spleen on rest imaging is of similar brightness compared to stress imaging).

\section{Statistical analysis}

Data are reported as mean $\pm S D$, tests are 2-tailed and parametric, based on Kolmogorov-Smirnov normalitychecks. Differences in individual characteristics were tested using Student's $t$-tests, paired within individuals (e.g. stress vs rest $\left.\mathrm{T} 1_{\text {spleen }}\right)$ and unpaired between groups (e.g. $\Delta \mathrm{T} 1_{\text {spleen }}$ in controls vs patients). Comparisons between $\geq 3$ data groups were assessed using analysis of variance (ANOVA) with Bonferronicorrected post-hoc method. Linear correlations were assessed using Pearson's correlation coefficient (R) and non-linear correlations were assessed using Spearman's rank correlation coefficient (rho). Intrascan variability and inter-observer reproducibility of rest/stress $T 1_{\text {spleen }}$ and $\Delta T 1_{\text {spleen }}$ were assessed using the Intra-class correlation coefficient (ICC), reporting 95\% confidence intervals. The performance of $\Delta \mathrm{T} 1_{\text {spleen }}$ for replicating splenic switch-off was assessed using receiver-operating characteristics (ROC) curves [21], reporting area-under-the-curve $(\mathrm{AUC} \pm \mathrm{SEM})$, and also sensitivity, specificity, diagnostic accuracy, positive predictive values (PPV) and negative predictive values (NPV), with 95\% confidence intervals (CI). All analyses were performed on single measures per-subject, using MedCalc 12.7.8 (MedCalc Software, Ostend, Belgium). $P<0.05$ denotes statistical significance.

\section{Results}

\section{Subject characteristics}

Subject characteristics are summarised in Table 1. All subjects experienced at least one adenosine-related symptoms (e.g. chest-tightness, dyspnoea, flushing) [13], and $>10 \mathrm{bpm}$ increase in heart rate (HR) during adenosine stress, compared to rest. Significant blood pressure response $(>10 \mathrm{mmHg}$ SBP decrease during stress) was observed in $50 \%$ of subjects.

Mean stress HR was lower in $1.5 \mathrm{~T}$ patients compared to other subjects, despite similar resting HR, likely due to more frequent beta-blocker and non-dihydropyridine calcium channel antagonist administration in these patients (all AF/CAD, Table 1).

\section{Stress and rest $\mathrm{T} 1_{\text {spleen }}$ in controls and patients}

In healthy controls, mean resting $\mathrm{T} 1_{\text {spleen }}$ values were 1102 $\pm 66 \mathrm{~ms}(1.5 \mathrm{~T})$ and $1352 \pm 114 \mathrm{~ms}(3 \mathrm{~T})$, which decreased significantly during adenosine stress at $1.5 \mathrm{~T}\left(\Delta \mathrm{T} 1_{\text {spleen }}\right.$ : $-40 \pm 25 \mathrm{~ms}, p<0.0001)$ and $3 \mathrm{~T}\left(\Delta \mathrm{T} 1_{\text {spleen }}:-43 \pm 31 \mathrm{~ms}\right.$, $p<0.0001)$. Patients had slightly lower resting $\mathrm{T} 1_{\text {spleen }}$ compared to controls at $1.5 \mathrm{~T}(1083 \pm 59 \mathrm{~ms}$ vs. $1102 \pm 66 \mathrm{~ms}$, $p=0.04$ ), and this pattern was more pronounced at $3 \mathrm{~T}$ $(1295 \pm 105 \mathrm{~ms}$ vs. $1352 \pm 114 \mathrm{~ms}, \mathrm{p}=0.01)$. Despite these observed resting $\mathrm{T} 1_{\text {spleen }}$ differences, $\Delta \mathrm{T} 1_{\text {spleen }}$ was comparable between patients and controls, at $1.5 \mathrm{~T}(-44 \pm 21 \mathrm{~ms}$ vs. $-40 \pm 25 \mathrm{~ms}, p=0.43)$ and $3 \mathrm{~T}(-44 \pm 26 \mathrm{~ms}$ vs. $-43 \pm$ $31 \mathrm{~ms}, p=0.93$; Table 2). In controls, there was a strong correlation between stress $\Delta \mathrm{T} 1_{\text {spleen }}$ (mean $-4.1 \pm 1.5 \%$ ) and $\Delta \mathrm{T} 1_{\text {myocardium }}$ (mean $5.9 \pm 1.8 \%$ ), $\mathrm{r}=-0.72, p<0.001$. See Additional file 1 for more details.

In pooled analysis, $\Delta \mathrm{T} 1_{\text {spleen }}$ did not appear to be significantly affected by field strength $(1.5 \mathrm{~T}$ vs. $3 \mathrm{~T}$ : $-43 \pm 22 \mathrm{~ms}$ vs. $-42 \pm 27 \mathrm{~ms}, \mathrm{p}=0.89$ ), gender (male vs. female: $-40 \pm$ $23 \mathrm{~ms}$ vs. $-47 \pm 28 \mathrm{~ms}, p=0.09)$, age $(\mathrm{R}=0.10, p=0.14$, range 21-89 years) or the type of cardiovascular diseases $(1.5 \mathrm{~T}$ CAD $-42 \pm 20 \mathrm{~ms}$ vs. $3 \mathrm{~T}$ CAD $-40 \pm 25 \mathrm{~ms}$ vs. AF $-46 \pm 22 \mathrm{~ms}$ vs. $\mathrm{HCM}-43 \pm 28 \mathrm{~ms}$ vs. AS $-43 \pm 21 \mathrm{~ms}$ vs. $\mathrm{DM}-43 \pm 32 \mathrm{~ms}, \mathrm{p}=0.54)$. In addition, $\Delta \mathrm{T} 1_{\text {spleen }}$ was not significantly affected by medication in patients (supplementary material in Additional file 2).

\section{$\mathrm{T} 1_{\text {spleen }}$ intra-scan variability}

Inter-slice intra-scan variability (assessable in 96 subjects) was within $\pm 19 \mathrm{~ms}$ for resting $\mathrm{T} 1_{\text {spleen }} \pm 18 \mathrm{~ms}$ for stress $\mathrm{T} 1_{\text {spleen }}$, and $\pm 10 \mathrm{~ms}$ for $\Delta \mathrm{T} 1_{\text {spleen. }}$ Re-analysis of the original ShMOLLI cohort (spleen visible in 9/10 cases), revealed an intra-slice intra-scan repeat variability of $\mathrm{T} 1_{\text {spleen }}$ of $\pm 9 \mathrm{~ms}, \mathrm{ICC}$ : 0.98 (95\% confidence interval 0.93 to 0.99$)[14] . \Delta \mathrm{T} 1_{\text {spleen }}$ was derived by a second independent blinded observer in 45 subjects (20 controls; 25 patients: 5 CAD, 5 AF, 5 DM, 5 AS, 5 HCM), which yielded an ICC of 0.87 (95\% confidence interval: 0.76 to 0.93 ). The Bland-Altman plot for inter-observer variability is shown in supplementary material (Additional file 3).

\section{Associations between splenic perfusion, $\mathrm{T} 1$ and rate pressure product (RPP)}

By semi-quantitative analysis, peak splenic perfusion SI $\left(\mathrm{SI}_{\text {spleen }}\right)$ decreased significantly with adenosine 
Table 1 Characteristics of study subjects: healthy controls and patients $(n=212)$

\begin{tabular}{|c|c|c|c|c|}
\hline & $\begin{array}{l}1.5 \mathrm{~T} \\
\text { Controls } \\
n=29\end{array}$ & $\begin{array}{l}1.5 \mathrm{~T} \\
\text { Patients } \\
n=75\end{array}$ & $\begin{array}{l}3 \mathrm{~T} \\
\text { Controls } \\
n=22\end{array}$ & $\begin{array}{l}3 \mathrm{~T} \\
\text { Patients } \\
n=86\end{array}$ \\
\hline Age (years) & $54 \pm 17$ & $65 \pm 9^{*}$ & $43 \pm 12$ & $60 \pm 14^{*}$ \\
\hline Men (\%) & $21(72)$ & $58(77)$ & $13(59)$ & $58(67)$ \\
\hline Body mass index $\left(\mathrm{kg} / \mathrm{m}^{2}\right)$ & $25 \pm 4$ & $28 \pm 5$ & $26 \pm 3$ & $28 \pm 4$ \\
\hline Hematocrit & $0.42 \pm 0.03$ & $0.43 \pm 0.03$ & $0.42 \pm 0.04$ & $0.42 \pm 0.03$ \\
\hline \multicolumn{5}{|l|}{ CMR hemodynamic data } \\
\hline Rest HR (bpm) & $66 \pm 11$ & $62 \pm 15$ & $62 \pm 12$ & $65 \pm 10$ \\
\hline Stress HR (bpm) & $96 \pm 15$ & $79 \pm 15^{*}$ & $95 \pm 12$ & $91 \pm 14$ \\
\hline Rest SBP (mmHg) & $133 \pm 21$ & $139 \pm 19$ & $127 \pm 14$ & $136 \pm 19$ \\
\hline Stress SBP (mmHg) & $127 \pm 19$ & $133 \pm 19$ & $122 \pm 16$ & $126 \pm 19$ \\
\hline Rest RPP (bpm.mmHg) & $8800 \pm 2200$ & $8600 \pm 2200$ & $7600 \pm 1700$ & $8700 \pm 2000$ \\
\hline Stress RPP (bpm.mmHg) & $12,200 \pm 2600$ & $10,500 \pm 2500^{*}$ & $12,000 \pm 2200$ & $11,700 \pm 2700$ \\
\hline Adenosine symptoms & $29(100)$ & $75(100)$ & $22(100)$ & $86(100)$ \\
\hline \multicolumn{5}{|l|}{ Co-morbidities } \\
\hline Current smoker & $3(10)$ & $2(3)$ & $2(9)$ & $12(14)$ \\
\hline Ex-smoker & $3(10)$ & $21(28)$ & $3(14)$ & $21(24)$ \\
\hline Hypertension & - & $28(37)$ & - & $24(28)$ \\
\hline Hyperlipidemia & - & $23(31)$ & - & $23(27)$ \\
\hline Stroke/TIA & - & $2(3)$ & - & $2(2)$ \\
\hline \multicolumn{5}{|l|}{ Medications } \\
\hline Aspirin & - & $36(48)$ & - & $35(41)$ \\
\hline Beta-blocker & - & $34(45)^{\#}$ & - & $19(22)$ \\
\hline ACEi/ARB & - & $36(48)$ & - & $39(45)$ \\
\hline Statin & - & $31(41)^{\#}$ & - & $44(51)$ \\
\hline Nitrates & - & $3(4)$ & & $4(5)$ \\
\hline CCB (non-DHP) & - & $13(17)^{\#}$ & - & $0(0)$ \\
\hline CCB (DHP) & & $4(5)$ & & $7(8)$ \\
\hline
\end{tabular}

Values are $n(\%)$ or mean \pm SD

Abbreviations: RPP rate pressure product, TIA transient ischemic attack, $A C E i$ angiotensin-converting enzyme inhibitors, $A R B$ angiotensin receptor blockers, $C C B$ calcium channel antagonist, DHP dihydropyridine

${ }^{*} p<0.05$ compared to controls of corresponding field strength (1.5 T or $3 \mathrm{~T}$ ). ${ }^{\#} p<0.05$ for comparisons between patient groups (1.5 T vs $3 \mathrm{~T}$ )

stress compared to rest, with no differences between controls and patients, or across field strengths (Table 3). $\Delta \mathrm{SI}_{\text {spleen }}$ correlated strongly with $\Delta \mathrm{T} 1_{\text {spleen }}$ $\left(\right.$ rho $=0.70, p<0.0001$, Fig. 2). In contrast, $\Delta \mathrm{SI}_{\text {spleen }}$ and $\Delta \mathrm{T} 1_{\text {spleen }}$ did not demonstrate significant correlations with stress-induced changes in RPP $(R=0.04, p$ $=0.60 ; R=0.06, p=0.38$, respectively).
Visual splenic switch-off assessment - relationships with perfusion, quantitative $\mathrm{T} 1_{\text {spleen }}$ and hemodynamic parameters

Subjects with visual splenic switch-off had greater stress $\Delta \mathrm{SI}_{\text {spleen }}$ and $\Delta \mathrm{T} 1_{\text {spleen }}$ values compared to those with no switch-off (Table 4 and Fig. 3). In contrast, there were no significant differences in stress-related

Table 2 Rest and stress splenic T1 in healthy controls and patients

\begin{tabular}{|c|c|c|c|c|c|c|}
\hline & $\begin{array}{l}1.5 \mathrm{~T} \\
\text { Controls }\end{array}$ & $\begin{array}{l}1.5 \mathrm{~T} \\
\text { Patients }\end{array}$ & $p$-value & $\begin{array}{l}3 \mathrm{~T} \\
\text { Controls }\end{array}$ & $\begin{array}{l}3 \mathrm{~T} \\
\text { Patients }\end{array}$ & $p$-value \\
\hline Rest $\mathrm{T} 1_{\text {spleen }}$ (ms) & $1102 \pm 66$ & $1083 \pm 59$ & 0.04 & $1352 \pm 114$ & $1295 \pm 105$ & 0.01 \\
\hline Stress $\mathrm{T} 1_{\text {spleen }}$ (ms) & $1061 \pm 68$ & $1039 \pm 55$ & 0.02 & $1308 \pm 114$ & $1253 \pm 112$ & 0.01 \\
\hline$\Delta T 1_{\text {spleen }}(\mathrm{ms})$ & $-40 \pm 25$ & $-44 \pm 21$ & 0.43 & $-43 \pm 31$ & $-44 \pm 26$ & 0.93 \\
\hline
\end{tabular}

$\Delta \mathrm{T} 1_{\text {spleen }}=$ Stress $T 1_{\text {spleen }}-$ Rest $T 1_{\text {spleen }}$ 
Table 3 Peak splenic perfusion signal intensity $\left(\mathrm{SI}_{\text {spleen }}\right)$ at rest and during adenosine stress $\left(\Delta S I_{\text {spleen }}\right)$ in healthy controls and patients

\begin{tabular}{|c|c|c|c|c|c|}
\hline & $\begin{array}{l}1.5 \mathrm{~T} \\
\text { Controls }\end{array}$ & $\begin{array}{l}1.5 \mathrm{~T} \\
\text { Patients }\end{array}$ & $\begin{array}{l}3 \mathrm{~T} \\
\text { Controls }\end{array}$ & $\begin{array}{l}3 \mathrm{~T} \\
\text { Patients }\end{array}$ & $p$-value \\
\hline Rest $S I_{\text {spleen }}(\mathrm{au})$ & $26 \pm 8$ & $24 \pm 11$ & $29 \pm 13$ & $27 \pm 13$ & 0.16 \\
\hline Stress $\mathrm{SI}_{\text {spleen }}(\mathrm{au})$ & $11 \pm 5$ & $11 \pm 5$ & $13 \pm 12$ & $12 \pm 8$ & 0.10 \\
\hline$\left.\Delta S\right|_{\text {spleen }}(\%)$ & $-58 \pm 23$ & $-54 \pm 22$ & $-56 \pm 28$ & $-52 \pm 30$ & 0.51 \\
\hline
\end{tabular}

Abbreviations: $A u$ arbitrary units

$P$-values derived using ANOVA with Bonferroni post-hoc method

haemodynamic changes (HR, SBP, RPP) between subjects with splenic switch-off and no switch-off (Table 4 and Fig. 3).

\section{ROC analysis of native $\Delta \mathrm{T} 1_{\text {spleen }}$ for replicating} gadolinium-based "splenic switch-off"

ROC analysis using visual splenic switch-off as reference standard (true positives = splenic switch-off, true negatives $=$ no switch-off) yielded an AUC of $0.90 \pm 0.05$ $\left(p<0.0001\right.$, Fig. 4). A $\Delta \mathrm{T} 1_{\text {spleen }}$ threshold of $\geq-30 \mathrm{~ms}$ during adenosine stress replicated visual splenic switch-off with a sensitivity of 90\% (95\% CI: $85-94 \%, p<0.0001$ ), specificity 88\% (95\% CI: 62-98\%, $p<0.0001$ ), diagnostic accuracy 90\% (95\% CI: 84-96\%, $p<0.0001$ ), PPV 98\% (95\% CI: $96-100 \%, p<0.0001)$ and NPV 42\% (95\% CI: $26-61 \%, p<0.0001)$.

\section{Discussion}

This proof of principle study demonstrated that $\mathrm{T} 1_{\text {spleen }}$ decreases significantly during adenosine stress compared to baseline. The magnitude of the stress-induced $\mathrm{T} 1_{\text {spleen }}$ response $\left(\Delta \mathrm{T} 1_{\text {spleen }}\right)$ is strongly correlated with splenic perfusion attenuation $\left(\Delta \mathrm{SI}_{\text {spleen }}\right)$. From a clinical viewpoint, a native $\Delta \mathrm{T} 1_{\text {spleen }}$ threshold of $\geq-30 \mathrm{~ms}$ accurately replicated the "splenic switch-off" sign with a high positive predictive value of $98 \%$ and offers the potential to assess adenosine stress adequacy before GBCA firstpass perfusion imaging. From a practical viewpoint, assessment of $\mathrm{T} 1_{\text {spleen }}$ takes $\sim 30 \mathrm{~s}$ (Fig. 5), which means it can be repeated as necessary "on-the-fly", to guide adenosine dosage up-titrations and optimize stress responses before injection of contrast agents (example protocol in Fig. 5). This pre-gadolinium approach may be advantageous over the retrospective and potentially gadolinium dose-sensitive splenic switch-off method for improving the quality of stress responses before firstpass perfusion imaging, which deserves further investigation in future studies to determine whether it decreases the number of false negative perfusion scans [6].

\section{Stress/rest $\mathrm{T} 1_{\text {spleen }}$ as a marker of adenosine stress response}

Patients had lower resting native $\mathrm{T} 1_{\text {spleen }}$ values compared to controls. This may be related to the presence of co-morbidities in patients, such as hypertension and peripheral vascular disease, which may induce peripheral vasoconstriction, with expected reductions in resting organ blood volumes and $\mathrm{T} 1_{\text {spleen }}$ values. This observation deserves further investigation in larger future studies. Native T1-relaxation times of tissues are prolonged by increased blood volume (i.e. water content) $[14,15,22]$. Adenosine causes splenic artery vasoconstriction and

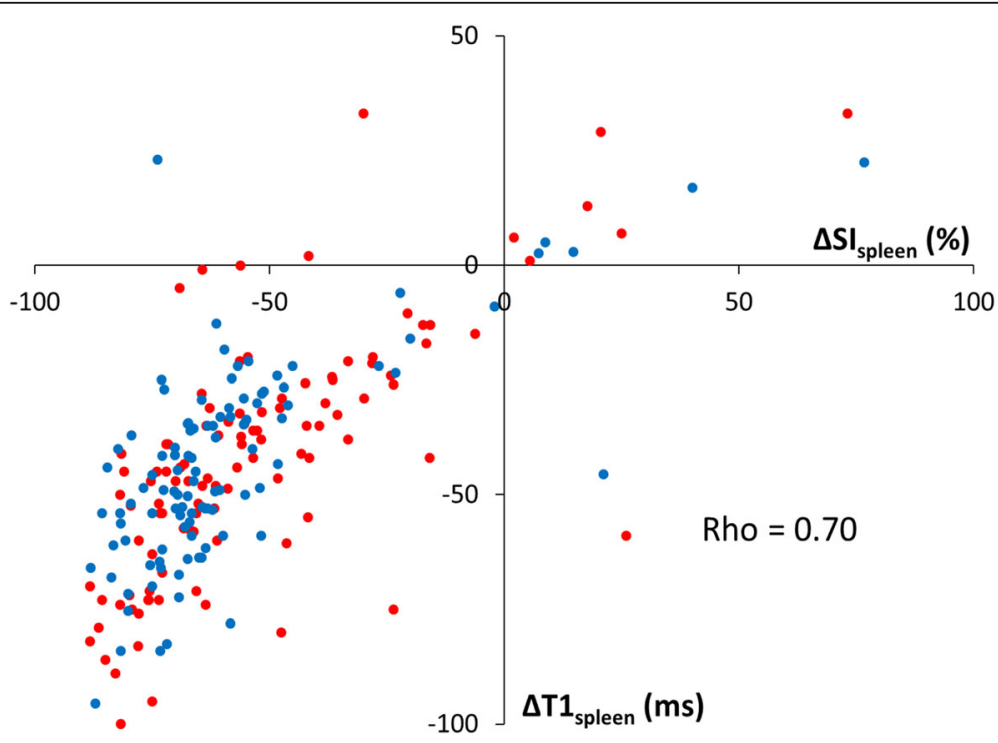

Fig. 2 Correlation between stress-induced reductions in peak splenic signal intensity $\left(\Delta S I_{\text {spleen }}\right)$ and splenic native $T 1\left(\Delta T 1_{\text {spleen }}\right)$. Pooled data of controls and patients at $1.5 \mathrm{~T}$ (blue) and $3 \mathrm{~T}(\mathrm{red})$, represented on per-subject basis $(n=212)$. Spearman's rank correlation coefficient $($ Rho $)=0.70, p<0.0001$ 
Table 4 Stress-induced changes in peak splenic perfusion signal intensity $\left(\Delta \mathrm{S} \mathrm{I}_{\text {spleen }}\right), \mathrm{T} 1\left(\Delta \mathrm{T} 1_{\text {spleen }}\right)$ and hemodynamic parameters for visually assessed "splenic switch-off" sign

\begin{tabular}{llll}
\hline & $\begin{array}{l}\text { Splenic } \\
\text { Switch-off }\end{array}$ & $\begin{array}{l}\text { No } \\
\text { Switch-off }\end{array}$ & -value \\
\hline All subjects $n=212$ & $196(92)$ & $16(8)$ & - \\
Healthy volunteers $n=51$ & $49(96)$ & $2(4)$ & - \\
$\quad$ Patients $n=161$ & $147(91)$ & $14(9)$ & - \\
$\Delta$ SI $_{\text {spleen }}(\%)$ & $-62 \pm 17$ & $17 \pm 29$ & $<0.0001$ \\
$\Delta$ T1 $_{\text {spleen }}(\mathrm{ms})$ & $-46 \pm 22$ & $-2 \pm 25$ & $<0.0001$ \\
Stress hemodynamic changes & & & \\
$\Delta$ heart rate (bpm) & $19 \pm 9$ & $20 \pm 12$ & 0.83 \\
$\Delta$ SBP (mm. Hg) & $-8 \pm 22$ & $-10 \pm 19$ & 0.76 \\
$\Delta$ RPP (bpm.mmHg) & $2800 \pm 2100$ & $2600 \pm 1700$ & 0.89 \\
\hline
\end{tabular}

Values are $n(\%)$ or mean \pm SD

Abbreviations: Bpm beats per minute, SBP systolic blood pressure, RPP rate pressure product

reduced blood volume [6-11], which shortens splenic T1-relaxation times. This is supported by our observation of significantly lower $\mathrm{T} 1_{\text {spleen }}$ during adenosine stress compared to rest, in both controls and patients. The stress $\Delta \mathrm{T} 1_{\text {spleen }}$ was not significantly affected by different field strengths, age, gender and cardiovascular diseases, likely reflecting reproducible T1-estimations in this study $[14,15,22]$.

The correlation between stress $\Delta \mathrm{T} 1_{\text {spleen }}$ and $\Delta \mathrm{T} 1_{\text {myo- }}$ cardium in normal controls suggests the vasoconstrictor effect of adenosine on the spleen is associated with vasodilatory effects in the myocardium. For the relationship between myocardial and splenic stress $\mathrm{T} 1$ in patients with cardiovascular disease, larger ongoing studies will offer reference ranges for $\Delta \mathrm{T} 1$ in disease, and resolve the separate effects of regional myocardial differences and medication on stress $\mathrm{T} 1$ reactivity.

The observed strong correlation between $\Delta \mathrm{T} 1_{\text {spleen }}$ and $\Delta \mathrm{SI}_{\text {spleen }}$ suggests that stress-induced changes in splenic blood volume are related to blood flow, which is regulated by alterations in the adenosine-mediated splenic arterial tone $[10,11]$. The lack of significant correlation between $\Delta \mathrm{SI}_{\text {spleen }}$ or $\Delta \mathrm{T} 1_{\text {spleen }}$ with rate pressure product is consistent with existing evidence showing dissociation between imaging and hemodynamic markers of stress response $[5,6]$, and further suggests that stress responses during clinical CMR cannot be reliably assessed using hemodynamic observations alone [5]. This deserves further investigation.

A threshold of $\geq 30 \mathrm{~ms}$ decrease in $\mathrm{T} 1_{\text {spleen }}$ replicated complete splenic switch-off with a high positive predictive value of $98 \%$. The intra-scan variability in $\mathrm{T} 1_{\text {spleen }}$ (inter-slice: $\pm 10 \mathrm{~ms}$; intra-slice: $\pm 9 \mathrm{~ms}$ ) was 3 -times less than this proposed threshold $\geq 30 \mathrm{~ms}$ drop, with excellent $\mathrm{T} 1$-fit as evident on quality control $\mathrm{R}^{2}$-maps, despite the lack of dedicated image optimization (e.g. shimming) over the spleen. For stress $\mathrm{T} 1_{\text {spleen }}$ responses $<30 \mathrm{~ms}$, further work is needed to determine whether adenosine dose-increments or waiting longer with the same infusion rate may improve the confidence of stress responses, and impact on diagnostic performance of stress CMR for the diagnosis of ischemia.

\section{Limitations and future directions}

This proof-of-concept study is based on ShMOLLI $\mathrm{T} 1_{\text {spleen }}$ values derived from short-axis slices planned for myocardial perfusion CMR imaging; the spleen was not visible in a small proportion of T1-maps ( 2\%), and future applications of splenic T1-mapping may benefit
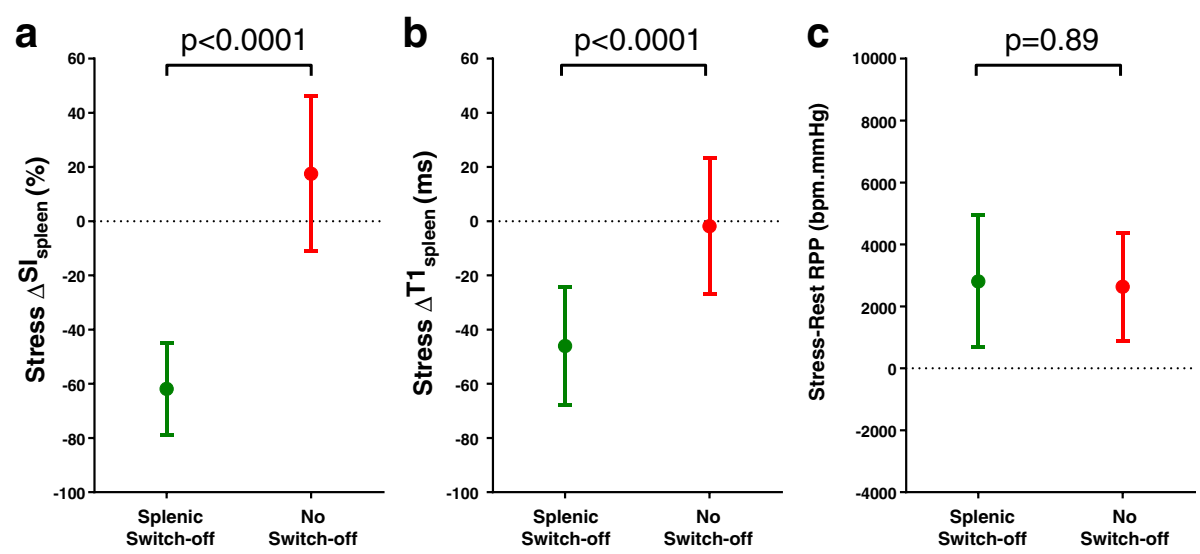

Fig. 3 Relations between different markers of stress adequacy. Subjects with the "splenic switch-off" sign had greater stress-induced reductions in a gadolinium-based splenic perfusion ( $\Delta \mathrm{SI}_{\text {spleen, }}$, same technique) and $\mathbf{b}$ gadolinium-free splenic $\mathrm{T} 1$ ( $\Delta \mathrm{T} 1_{\text {spleen, }}$ different technique) compared to subjects with no switch-off. There was no difference in stress-induced $\mathbf{c}$ hemodynamic changes in rate pressure product (RPP) between the splenic switch-off and the no switch-off subjects. Data are mean \pm 1 SD 


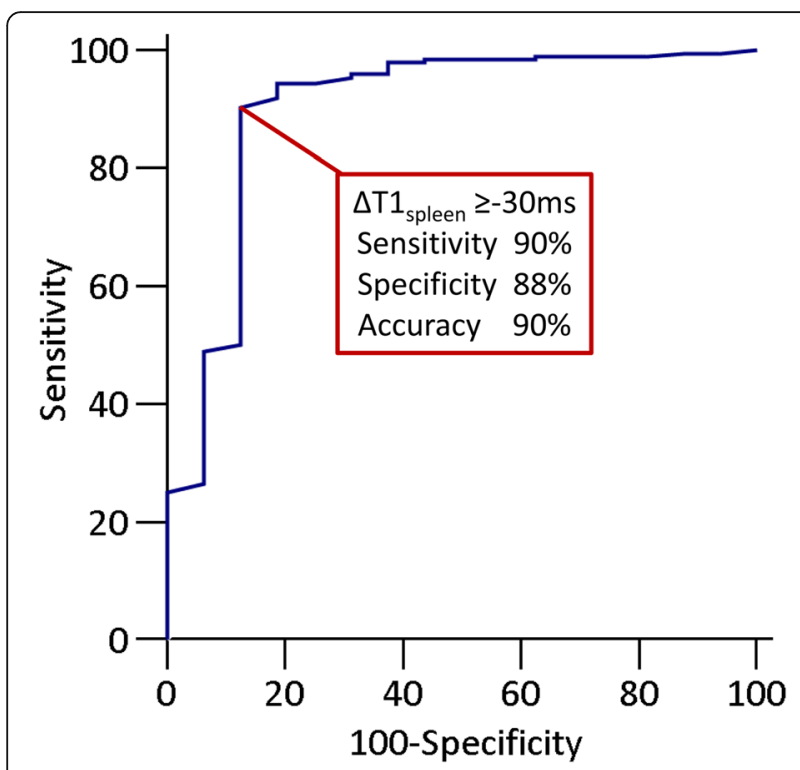

Fig. 4 ROC curves of native $\Delta \mathrm{T} 1_{\text {spleen }}$ for replicating the gadolinium-based "splenic switch-off" sign. A $\Delta T 1_{\text {spleen }}$ threshold of $\geq-30 \mathrm{~ms}$ replicated the "splenic switch-off" sign (AUC 0.90 \pm 0.05 , $p<0.0001$ ), with high sensitivity $90 \%$, specificity $88 \%$ and diagnostic accuracy $90 \%$

from a dedicated image planned through the spleen. Rapid on-scanner T1-map reconstructions, with the immediate availability of goodness-of-fit measures (such as $\mathrm{R}^{2}$-maps), are imperative to enable practical "on-the-fly" repetition of reliable $\mathrm{T} 1_{\text {spleen }}$ estimations to guide stress response optimization (Fig. 5). Given the overall excellent $\mathrm{R}^{2}$-maps over the spleen and the narrow $T 1_{\text {spleen }}$ ranges obtained, data in this study suggest that stress/rest splenic T1-mapping can be feasibly included in CMR protocols without major technical adjustments. Practical in-vivo T1estimations are method-dependent, and demonstrate increasingly discrepant heart rate dependencies at longer T1-values [23]. Therefore, results achieved with ShMOLLI, in particular the splenic T1-thresholds replicating splenic switch-off, should be interpreted with care before directly translating to other T1-mapping techniques. Choosing methods that can withstand dynamic HRvariations and tachycardia without significant HRdependencies is therefore paramount when performing stress-T1 studies. The gadolinium-based splenic switchoff sign is only seen with non-selective adenosine receptor agonists (dipyridamole and adenosine), but was absent with cardio-selective vasodilators (e.g. regadenoson) or inotropic agents (e.g. dobutamine) [6]. Further work is needed to elucidate stress $\mathrm{T} 1_{\text {spleen }}$ responses using pharmacological agents other than adenosine and during physical exercise. Patients in this study were unselected for diseases known to affect splenic blood volumes, e.g. venous portal hypertension, hematological malignancies and systemic inflammation; thus, further studies to characterize the effects of these diseases on $\mathrm{T} 1_{\text {spleen }}$ will help to determine the general applicability of this technique. While we identified a cut-off of $\geq 30 \mathrm{~ms}$ drop in $\mathrm{T} 1_{\text {spleen }}$ during stress for replicating complete splenic switch-off, the clinical utility of this threshold for detecting true stress adequacy needs to be validated against false-negative perfusion scans, determined by comparison to invasive coronary angiography and pressure-wire based assessments of functional ischemia, such as fractional flow reserve. This is topic of ongoing work.

\section{Conclusions}

Adenosine stress and rest splenic T1-mapping is a novel method for assessing stress responses, independent of conventional hemodynamic parameters. It

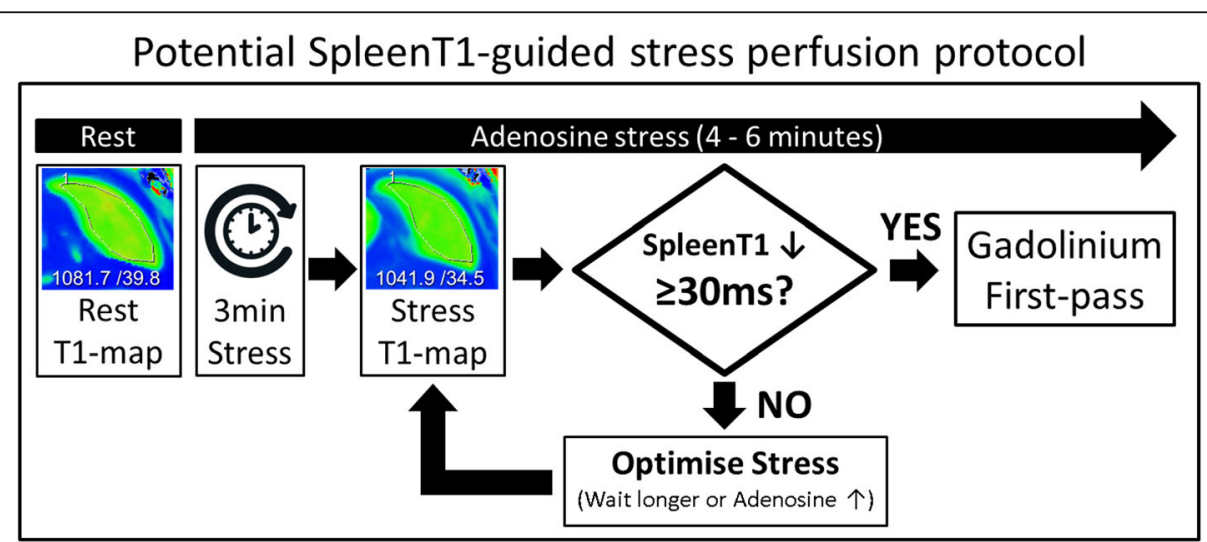

Fig. 5 Potential splenic $\Delta T 1_{\text {spleen }}$-guided protocol for real-time assessment and optimization of stress adequacy before gadolinium perfusion. Practical $\mathrm{T1} 1_{\text {spleen }}$ assessment using ShMOLLI typically takes around $30 \mathrm{~s}$ : breath-hold instructions (5 s), T1-map acquisition over 9-heart-beats ( $10 \mathrm{~s}$, shorter with higher stress heart rates), on-screen image reconstruction (5-10 s), splenic-ROI placement directly on CMR console screen by the operator $(5 \mathrm{~s})$ followed by immediate display of $\mathrm{T} 1_{\text {spleen }} / \mathrm{SD}$ estimations (as indicated). The ability of this protocol to improve the quality of stress responses deserves validation in future studies 
enables prediction of the visual "splenic switch-off" sign without the need for gadolinium, and correlates well to changes in splenic signal intensity during stress/rest perfusion imaging. $\Delta \mathrm{T} 1_{\text {spleen }}$ holds promise to facilitate optimization of stress responses before gadolinium first-pass perfusion CMR.

\section{Additional files}

Additional file 1: Figure S1. Description of data: Correlation between adenosine stress $\Delta T 1_{\text {spleen }}$ and $\Delta T 1_{\text {myocardium }}$ in 51 healthy controls. Data are presented per-subject. (DOCX $26 \mathrm{~kb}$ )

Additional file 2: Table S1. Description of data: Effect of medication on $\Delta T 1_{\text {spleen }}$ in patients with cardiovascular disease. ACE: angiotensin converting enzyme; ARB: angiotensin reception blocker; CCB: calcium channel blockers; DHP: dihydropyridine. (DOCX $13 \mathrm{~kb}$ )

Additional file 3: Figure S2. Description of data: Bland Altman plot of $\Delta T 1_{\text {spleen }}$ estimation by 2 independent blinded observers. (DOCX $38 \mathrm{~kb}$ )

\section{Abbreviations}

CAD: Coronary artery disease; CMR: Cardiovascular magnetic resonance; ECG: Electrocardiogram; GBCA: Gadolinium-based contrast agents; ROC: Receiver-operating characteristics; ShMOLLI: Shortened modified look-locker inversion recovery; SI spleen: Peak splenic signal intensity; $\mathrm{T} 1_{\text {spleen: }}$ : Mean native splenic $\mathrm{T} 1$ values

\section{Acknowledgements}

Not applicable.

\section{Funding}

This study was supported in part by the National Institute for Health Research (NIHR) Oxford Biomedical Research Centre at The Oxford University Hospitals, University of Oxford, UK. AL is funded by a British Heart Foundation Clinical Research Training Fellowship grant (FS/15/11/31233). RSW, SN, SKP and VMF acknowledge support from the British Heart Foundation Centre of Research Excellence, Oxford, UK (RE/08/004 to RSW). AG is an employee of Siemens Healthcare $\mathrm{GmbH}$.

Funding bodies had no influence on the design of the study and collection, analysis, and interpretation of data and in writing the manuscript.

\section{Availability of data and materials}

The datasets during and/or analysed during the current study available from the corresponding author on reasonable request.

\section{Authors' contributions}

All authors made appropriate contributions according to the ICMJE guidance, and as such have read and approved the final manuscript. All authors take public responsibility for appropriate portions of the manuscript content; and agree to be accountable in ensuring that questions related to the accuracy or integrity of the work are appropriately investigated and resolved. In addition, author specific contributions to the study are listed below: AL contributed to study conception and design, subject recruitment, data acquisition, analysis and interpretation; drafting of manuscript and revisions. RSW contributed to subject recruitment, data acquisition, analysis, interpretation and manuscript revisions. RA, MM, EL contributed to subject recruitment, data acquisition and manuscript revisions. AG contributed to giving technical advice on T1-mapping and manuscript revisions. MP, GK, JCF, RKK, KMC contributed to subject recruitment and manuscript revisions. SN contributed to study conception and design, data interpretation, study supervision and manuscript revisions. SKP contributed to study conception and design, data analysis, interpretation, study supervision and manuscript revisions. VMF contributed to study conception and design, subject recruitment, data acquisition, analysis, interpretation, study supervision and manuscript revisions.

\section{Competing interests}

SKP has patent authorship rights for U.S. patent 9285446 B2. Systems and methods for shortened look locker inversion recovery (Sh-MOLLI) cardiac gated mapping of T1. Granted March 15, 2016. All rights transferred to Siemens Medical.

All other authors have no relationships relevant to the contents of this paper to disclose.

\section{Consent for publication}

All subjects gave written informed consent for publication.

\section{Ethics approval and consent to participate}

This study was approved by the South Central Oxford A Health Research Authority (formerly known as Oxfordshire Research Ethics Committee A) based at Bristol HRA Centre, Level 3, Block B, Whitefriars, Lewins Mead, Bristol, BS1 2NT, UK. All subjects gave written informed consent.

\section{Author details}

${ }^{1}$ Oxford Centre for Clinical Magnetic Resonance Research (OCMR), Division of Cardiovascular Medicine, Radcliffe Department of Medicine, University of Oxford, Oxford, UK. ${ }^{2}$ Siemens Healthcare GmbH, Erlangen, Germany. ${ }^{3}$ Department of Cardiothoracic Surgery, John Radcliffe Hospital, Oxford, UK. ${ }^{4}$ Oxford Heart Centre, John Radcliffe Hospital, Oxford, UK. ${ }^{5}$ Division of Cardiovascular Medicine, Radcliffe Department of Medicine, University of Oxford, Oxford, UK.

Received: 8 October 2016 Accepted: 20 December 2016 Published online: 13 January 2017

\section{References}

1. Salerno M, Beller GA. Noninvasive assessment of myocardial perfusion. Circ cardiovasc imaging. 2009;2(5):412-24

2. Hamon M, Fau G, Nee G, Ehtisham J, Morello R, Hamon M. Meta-analysis of the diagnostic performance of stress perfusion cardiovascular magnetic resonance for detection of coronary artery disease. J cardiovasc magn reson. 2010;12(1):29

3. Jaarsma C, Leiner T, Bekkers SC, Crijns HJ, Wildberger JE, Nagel E, Nelemans PJ, Schalla S. Diagnostic performance of noninvasive myocardial perfusion imaging using single-photon emission computed tomography, cardiac magnetic resonance, and positron emission tomography imaging for the detection of obstructive coronary artery disease: a meta-analysis. J am coll cardiol. 2012;59(19):1719-28.

4. Kidambi A, Sourbron S, Maredia N, Motwani M, Brown JM, Nixon J, Everett CC, Plein S, Greenwood JP. Factors associated with false-negative cardiovascular magnetic resonance perfusion studies: a clinical evaluation of magnetic resonance imaging in coronary artery disease (CE-MARC) substudy. J magn reson imaging. 2016;43(3):566-73.

5. Mishra RK, Dorbala S, Logsetty G, Hassan A, Heinonen T, Schelbert HR, Di Carli MF, R. investigators. Quantitative relation between hemodynamic changes during intravenous adenosine infusion and the magnitude of coronary hyperemia: implications for myocardial perfusion imaging. J am coll cardiol. 2005:45(4):553-8.

6. Manisty C, Ripley DP, Herrey AS, Captur G, Wong TC, Petersen SE, Plein S, Peebles C, Schelbert EB, Greenwood JP, Moon JC. Splenic switch-off: a tool to assess stress adequacy in adenosine perfusion cardiac MR imaging. Radiology. 2015;276(3):732-40.

7. Flamm SD, Taki J, Moore R, Lewis SF, Keech F, Maltais F, Ahmad M, Callahan R, Dragotakes S, Alpert N, et al. Redistribution of regional and organ blood volume and effect on cardiac function in relation to upright exercise intensity in healthy human subjects. Circulation. 1990;81(5):1550-9.

8. Froelich JW, Strauss HW, Moore RH, Mckusick KA. Redistribution of visceral blood volume in upright exercise in healthy volunteers. J nucl med. 1988; 29(10):1714-8.

9. Gates GF, Ames AW. Splenic "disappearance" during gated exercise nuclear angiocardiography. Clin nucl med. 1986;11(10):683-7.

10. Yang XP, Chiba S. Endothelium-released adenosine triphosphate contributes to vasoconstrictor responses to periarterial nerve stimulation in isolated, perfused canine splenic arteries. Heart vessels. 1998;13(5):256-61.

11. Fozard JR, Milavec-Krizman M. Contraction of the rat isolated spleen mediated by adenosine A1 receptor activation. Br j pharmacol. 1993;109(4): 1059-63. 
12. Fernandes JL, Fioravante LA, Mazo PE, Greiser A, Strecker R. Use of T2 maps for rapid prediction of stress effectiveness before the injection of contrast in myocardial perfusion studies at 3.0T. J cardiovasc magn reson. 2016;18 Suppl 1:Q53.

13. Karamitsos TD, Ntusi NA, Francis JM, Holloway CJ, Myerson SG, Neubauer S. Feasibility and safety of high-dose adenosine perfusion cardiovascular magnetic resonance. J cardiovasc magn reson. 2010;12:66.

14. Piechnik SK, Ferreira VM, Dall'armellina E, Cochlin LE, Greiser A, Neubauer S, Robson MD. Shortened modified look-locker inversion recovery (ShMOLLI) for clinical myocardial T1-mapping at 1.5 and $3 \mathrm{~T}$ within a 9 heartbeat breathhold. J cardiovasc magn reson. 2010;12:69.

15. Liu A, Wijesurendra RS, Francis JM, Robson MD, Neubauer S, Piechnik SK, Ferreira VM. Adenosine stress and rest T1 mapping Can differentiate between ischemic, infarcted, remote, and normal myocardium without the need for gadolinium contrast agents. JACC cardiovasc imaging. 2016;9(1):27-36

16. Mahmod M, Piechnik SK, Levelt E, Ferreira VM, Francis JM, Lewis A, Pal N, Dass S, Ashrafian H, Neubauer S, Karamitsos TD. Adenosine stress native T1 mapping in severe aortic stenosis: evidence for a role of the intravascular compartment on myocardial T1 values. J cardiovasc magn reson. 2014;16:92.

17. Ferreira VM, Piechnik SK, Dall'armellina E, Karamitsos TD, Francis JM, Choudhury RP, Friedrich MG, Robson MD, Neubauer S. Non-contrast T1-mapping detects acute myocardial edema with high diagnostic accuracy: a comparison to T2-weighted cardiovascular magnetic resonance. J cardiovasc magn reson. 2012;14:42.

18. Ferreira VM, Piechnik SK, Dall'armellina E, Karamitsos TD, Francis JM, Ntusi N, Holloway C, Choudhury RP, Kardos A, Robson MD, Friedrich MG, Neubauer S. T1 mapping for the diagnosis of acute myocarditis using CMR: comparison to T2-weighted and late gadolinium enhanced imaging. JACC cardiovasC imaging. 2013;6(10):1048-58.

19. Ferreira VM, Piechnik SK, Dall'armellina E, Karamitsos TD, Francis JM, Ntusi N, Holloway C, Choudhury RP, Kardos A, Robson MD, Friedrich MG, Neubauer S. Native T1-mapping detects the location, extent and patterns of acute myocarditis without the need for gadolinium contrast agents. J cardiovasc magn reson. 2014;16:36.

20. Ferreira VM, Wijesurendra RS, Liu A, Greiser A, Casadei B, Robson MD, Neubauer S, Piechnik SK. Systolic ShMOLLI myocardial T1-mapping for improved robustness to partial-volume effects and applications in tachyarrhythmias. J cardiovasc magn reson. 2015;17:77.

21. Delong ER, Delong DM, Clarke-Pearson DL. Comparing the areas under two or more correlated receiver operating characteristic curves: a nonparametric approach. Biometrics. 1988;44(3):837-45.

22. Piechnik SK, Ferreira VM, Lewandowski AJ, Ntusi NA, Banerjee R, Holloway C, Hofman MB, Sado DM, Maestrini V, White SK, Lazdam M, Karamitsos T, Moon JC, Neubauer S, Leeson P, Robson MD. Normal variation of magnetic resonance $\mathrm{T} 1$ relaxation times in the human population at $1.5 \mathrm{~T}$ using ShMOLLI. J cardiovasc magn reson. 2013;15:13.

23. Kellman P, Hansen MS. T1-mapping in the heart: accuracy and precision. J cardiovasc magn reson. 2014;16:2

\section{Submit your next manuscript to BioMed Central and we will help you at every step:}

- We accept pre-submission inquiries

- Our selector tool helps you to find the most relevant journal

- We provide round the clock customer support

- Convenient online submission

- Thorough peer review

- Inclusion in PubMed and all major indexing services

- Maximum visibility for your research

Submit your manuscript at www.biomedcentral.com/submit
Biomed Central 